\title{
DUST AND GAS IN SHELLS AROUND HERBIG Ae/Be
}

\section{STARS}

\author{
N.V. VOSHCHINNIKOV \\ $M P G$ "Dust in Star-Forming Regions", Jena, Germany and \\ Astronomical Observatory, St. Petersburg University, St. Petersburg, Russia \\ and \\ V.B. IL'IN, A.F. KHOLTYGIN \\ Astronomical Observatory, St. Petersburg University, St. Petersburg, Russia
}

Herbig Ae/Be (HAEBE) stars are young objects with strong infrared excesses, variable brightness, intrinsic polarization and complex spectral line profiles. Almost all of these features are due to the presence of circumstellar (CS) shells. We consider the group of HAEBE stars with non-periodic Algollike minima (UX Ori, WW Vul, etc.). They have irregular brightness drops by 2-3 mag in the visual, accompanied by an increase of linear polarization.

Model. We use the model with variable CS extinction (Grinin, 1988; Grinin et al., 1991). The unusual photometric behaviour of these stars is explained by the presence of very young protoplanetary systems most likely seen edge-on. Many CS dust clumps of different size and mass rotate around such a star and screen off its radiation from an observer from time to time. The shape of the CS shell is assumed to be spheroidal with semiaxes ratio $A / B$. We consider various silicate-graphite mixtures of dust grains with a power-law size distribution.

Single Scattering Modelling. Using the observations of WW Vul in two deep minima, a model of the dust shell around this star has been constructed (see Voshchinnikov and Grinin, 1991 for details). In both cases, the CS shell has similar characteristics (see Table I), but the properties of CS clumps screening the star are different. The absence of small grains may be explained by their sweeping out of the shell by radiation pressure (Il'in and Voshchinnikov, 1993). If the outer shell radius is at $A=140 \mathrm{AU}$, the mass of dust grains is $\sim 10^{25} \mathrm{~g}$. Apparently, only this part of the shell gives the main contribution to the scattered radiation and polarization. Taking into account the total duration of minima, the radius of the clouds $(\approx 0.2-0.7$ AU) has been estimated. The clouds have orbits with large eccentricity and major semiaxes $\geq 10 \mathrm{AU}$. In order to investigate the cloud dynamics in detail, it is necessary to know their radial velocities which can be obtained from observations of the gas absorption lines originating in the clumps.

Multiple Scattering Modelling. On the basis of a new modification of the Monte Carlo approach, a numerical code for polarized radiation transfer in non-spherical CS shells has been developed (see Voshchinnikov et al., 1993 
TABLE I

Parameters of dust shell around WW Vul

\begin{tabular}{ccccccc}
\hline Parameter & $a_{-}, \mu \mathrm{m}$ & $a_{+}, \mu \mathrm{m}$ & $q$ & $n_{\mathrm{Si}} / n_{\mathrm{C}}$ & $A / B$ & $\tau_{90}^{\text {ext }}(0.36 \mu \mathrm{m})$ \\
\hline Model & 0.055 & 0.25 & 5.0 & 0.25 & 3 & 0.55 \\
Standard MRN mixture & 0.005 & 0.25 & 3.5 & 1.07 & & \\
\hline
\end{tabular}

and Voshchinnikov and Karjukin, 1993 for details).

Our calculations for the model of WW Vul show that there are polarization reversals at $\lambda \approx 1600 \AA$ and $1900 \AA$. They are caused by the peculiarity of the dust mixture. The radiation scattered by dust grains may be up to $20-30 \%$ of the total radiation from the system: star + envelope and is the most significant at $\lambda \approx 2000-3000 \AA$. The fraction of scattered radiation becomes smaller in the red part of the spectrum. Since the thermal infrared radiation of grains begins to distort the spectrum of HAEBE stars only at $\lambda>1 \mu \mathrm{m}$, we can conclude that the influence of a CS dust shell on the observed radiation is minimal around $\lambda \approx 1 \mu \mathrm{m}$.

Ionization Structure Modelling. The ionization balance in the outer layers of CS shells has been considered assuming that the sources of gas ionization are: stellar photosphere, stellar chromosphere, and H II region (Kholtygin et $a l ., 1993)$. We made calculations of column densities $(N)$ of various ions as well as equivalent widths $\left(W_{\lambda}\right)$ of the $\mathrm{D}_{1}, \mathrm{D}_{2}$ lines of $\mathrm{NaI}$ and $\mathrm{H}, \mathrm{K}$ lines of $\mathrm{Ca}$ II which are most suitable to be observed during the minima of HAEBE stars. It was shown that the values of $N$ and $W_{\lambda}$ depend only weakly on the characteristics of the H II region and the CS clumps. The strongest effect is produced by the variations of the gas to dust ratio, element depletion, and velocity of the large scale motion in the clumps. The calculated values of $W_{\lambda}$ for $\mathrm{NaI}$ and $\mathrm{Ca}$ II lines definitively indicate that they may be observed with current big telescopes during minima of the bright HAEBE stars.

Thus, the theoretical basis for the investigations of protoplanetary disks around Herbig $\mathrm{Ae} / \mathrm{Be}$ stars is created. The photometric, polarimetric, and spectral observations of these stars at various brightness levels can be used to obtain more information on the process of planet formation.

\section{References}

Grinin, V.P.: 1988, Sov. Astron. Lett. 14, 65.

Grinin, V.P., Kiselev, N.N., Minikulov, N.Kh., Chernova, G.P., and Voshchinnikov, N.V.: 1991, Astrop. Sp. Sci. 186, 283.

Il'in, V.B. and Voshchinnikov, N.V.: 1993, Astron. Zh. 70, 721.

Kholtygin, A.F., Il'in, V.B., and Voshchinnikov, N.V.: 1993, Astrop. Sp. Sci., in prep.

Voshchinnikov, N.V. and Grinin, V.P.: 1991, Astrofizika 34, 181.

Voshchinnikov, N.V., Grinin, V.P., and Karjukin, V.V.: 1993, $A \& A$, submitted.

Voshchinnikov, N.V. and Karjukin, V.V.: 1993, A\&A Supp., submitted. 